\title{
Partial Small Intestine Resection
}

National Cancer Institute

\section{Source}

National Cancer Institute. Partial Small Intestine Resection. NCI Thesaurus. Code C51512.

Surgical removal of part of the small intestine. 